\title{
Thermal Analysis of Organic Medicinals. I. Detection of the Molecular Compound Formation by the Differential Thermal Analysis and by the Differential Scanning Calorimetry
}

\author{
Keiji Sekiguchi, Ikuzo Himuro, Isamu Horikoshr, \\ Toshiko Tsukada, Takako Okamoto, ${ }^{1 a}$ \\ and ToMOHisa YotsUYANAgi ${ }^{1 b}$ ) \\ School of Pharmaceutical Sciences, Kitasato Memorial University, ${ }^{1 a}{ }^{2}$ and Faculty \\ of Pharmaceutical Sciences, University of Tokyo1b)
}

(Received September 17, 1968)

\begin{abstract}
The differential thermal analysis (DTA) and the differential scanning calorimetry (DSC) were carried out with various two-component mixtures of organic materials prepared by mechanical mixing, by solidification after fusion and by evaporation of the solution of components. As was expected, when the components did not form a molecular compound, no distinct difference in the thermograms were observed between the kinds of samples except that in the ones for the simple physical mixture, additional small peaks due to polymorphic transition appeared occasionally.

On the contrary, the method of sample preparation exerted marked influence on the result, if the molecular compound was formed between components. Usually, the evaporated mixture solidifies in the state of stable equilibrium; therefore, the DTA and DSC curves were the same as that for the mixture of the isolated compound and one of the components. In the case of the physical mixture which is in the metastable state, the curves showed characteristic peaks of the metastable fusion (endothermic), and of the subsequent compound formation and solidification (exothermic). Thus, by applying DTA or DSC to the physical mixture, a simple method was developed for detecting the molecular compound between organic materials. Also, the heat of formation of the molecular compound could be obtained by reducing the total area of the DSC pealss for the isolated compound from that for the corresponding physical mixture.
\end{abstract}

The differential thermal analysis (DTA) has become a popular means of investigation; however, its application to pharmaceutical problems are not sufficiently explored. Previously, the authors suggested that formation of the molecular compound would be detected by DTA and gave one example concerning the interaction between aminopyrine and barbital. ${ }^{2)}$ In the present investigation, the possibility was examined with various two-component mixtures of organic compounds. Thus, a simple method for detecting the molecular compound was developed.

\section{Experimentals}

Apparatus-1) DTA-Apparatus: The heating bath, cells and the recording system were the same as before. ${ }^{2}$ The electric circuit was modified as shown in Fig. 1. Very fine powder of KCI (G.R. grade) made by freeze-drying was used as the reference. Appearance or disappearance of liquid or solid was observed visually during the course of measurement.

2) Differential Scanning Calorimeter: Perkin Elmer Model DSC 1B Differential Scanning Calorimeter was used.

Materials_-The J.P. drugs were used without further purification. The rest of the materials were purified by recrystallization.

Sample Preparation-1) Molecular Compounds: The mixture containing the two components in proportion to the composition of the molecular compound was added into the solution just saturated with both the molecular compound and either one of the components and was then dissolved by warming. After filtration, crystals of the molecular compound were separated out very gradually.

1) Location: a) Shirokane-Sankocho, Minatoku, Tokyo; b) Hongo, Bunkyoku, Tokyo.

2) K. Sekiguchi, T. Yotsuyanagi, and S. Mikami, Chem. Pharm. Bull. (Tokyo), 12, 994 (1964). 
2) Physical Mixtures: The two components or the molecular compound and one of its components were mixed lightly or thoroughly in an agate mortar.

3) Fused Mixtures: The physical mixture in the sample cell was inserted with a thermistor, and was. heated in a silicon bath. After the mixture was completely fused, the melt was allowed to solidify.

4) Evaporated Mixtures: A portion of the physical mixture was dissolved in a solvent. The solvent. was then evaporated to dryness on a water bath. The residue was solidified by keeping it in an electric drying oven at a temperature lower than the eutectic point but higher than the metastable eutectic point of the sample mixture. The solid mass was ground to a fine powder.

Phase Diagrams — The phase diagram of the system of antipyrine and phenobarbital was reconstructed by the modified thaw-melt method, ${ }^{3}$ ) since the one by Pfeifer and Seydel was imperfect. ${ }^{4}$. Diagrams of other systems were taken from literature, but the thaw points of them were corrected by the above method, if necessary.
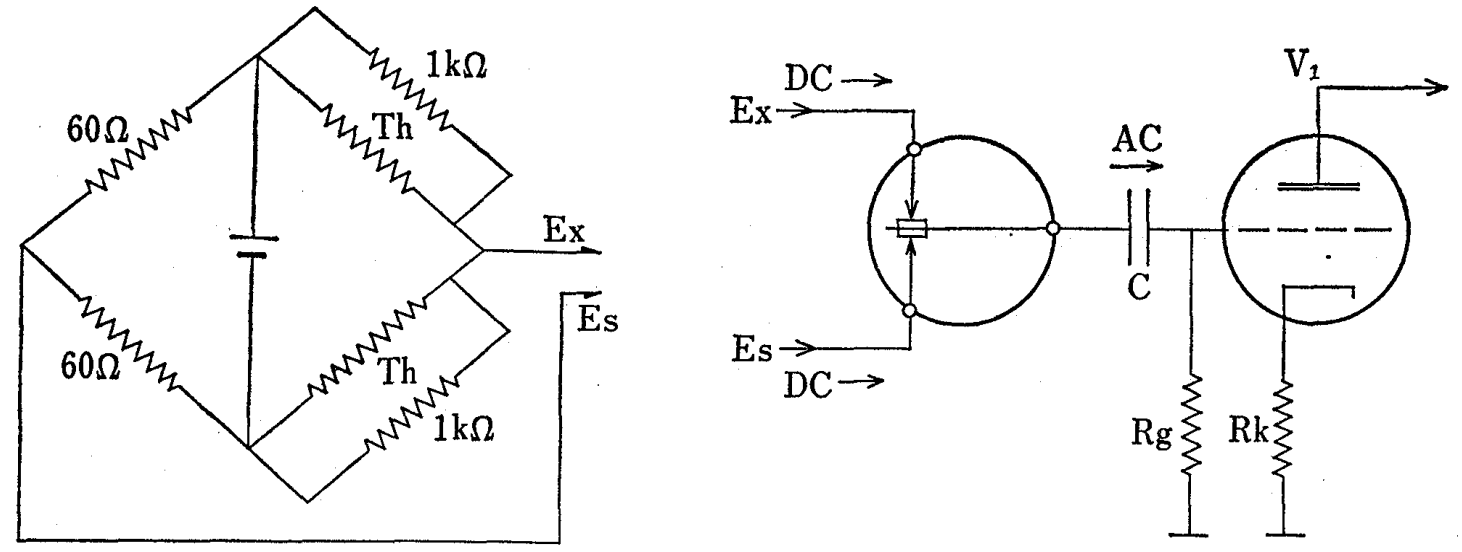

Fig. 1. Electric Circuit of DTA Apparatus

[A] Bridge Circuit

[B] Chopper Circuit

\section{Results and Discussion}

When the components do not form a molecular compound, the DTA and DSC curves for the three kinds of sample mixtures are almost the same as shown in Fig. 2.5) If one or both of the components has polymorphic forms, additional peaks corresponding to transition appear in the curve for the physical mixture. On the other hand, when the fused and the evaporated samples are applied to the measurement immeadiately after preparation of them, these peaks disappear practically or become indistinct (Fig. 3).

If a molecular compound is formed between components, considerable difference in the DTA and DSC curves was noted by the method of sample preparation. Since the mixture prepared by evaporation of the solvent solidifies usually in a stable equilibrium state, the curve becomes similar to that of the molecular compound or of its mixture with either one of the components; accordingly, the pattern of the curve and the positions of peaks are fixed. Although in most cases, the molecular compound is formed completely in the fused mixture, it sometimes happens that the mixture solidifies in a metastable or an unstable state. As the result, the DTA and DSC curves often differ considerably from that of the stable mixture (Fig. 4, 8). For this reason, the fused mixture is not suitable for the purpose of detecting the interaction between components.

In the case of the physical mixture which is always in the metastable state, the DTA and DSC curves show characteristic peaks due to the metastable fusion (endothermic), and the subsequent molecular compound formation and solidification (exothermic). Based on the

3) K. Sekiguchi, Y. Ueda, and Y. Nakamori, Chem. Pharm. Bull. (Tokyo), 11, 1108 (1963).

4) P. Pfeifer and R. Seydel, Z. Physical. Chem., 17, 107 (1928).

5) K. Sekiguchi, N. Obi, and Y. Ueda, Chem. Pharm. Bull. (Tokyo), 12, 134 (1964). 

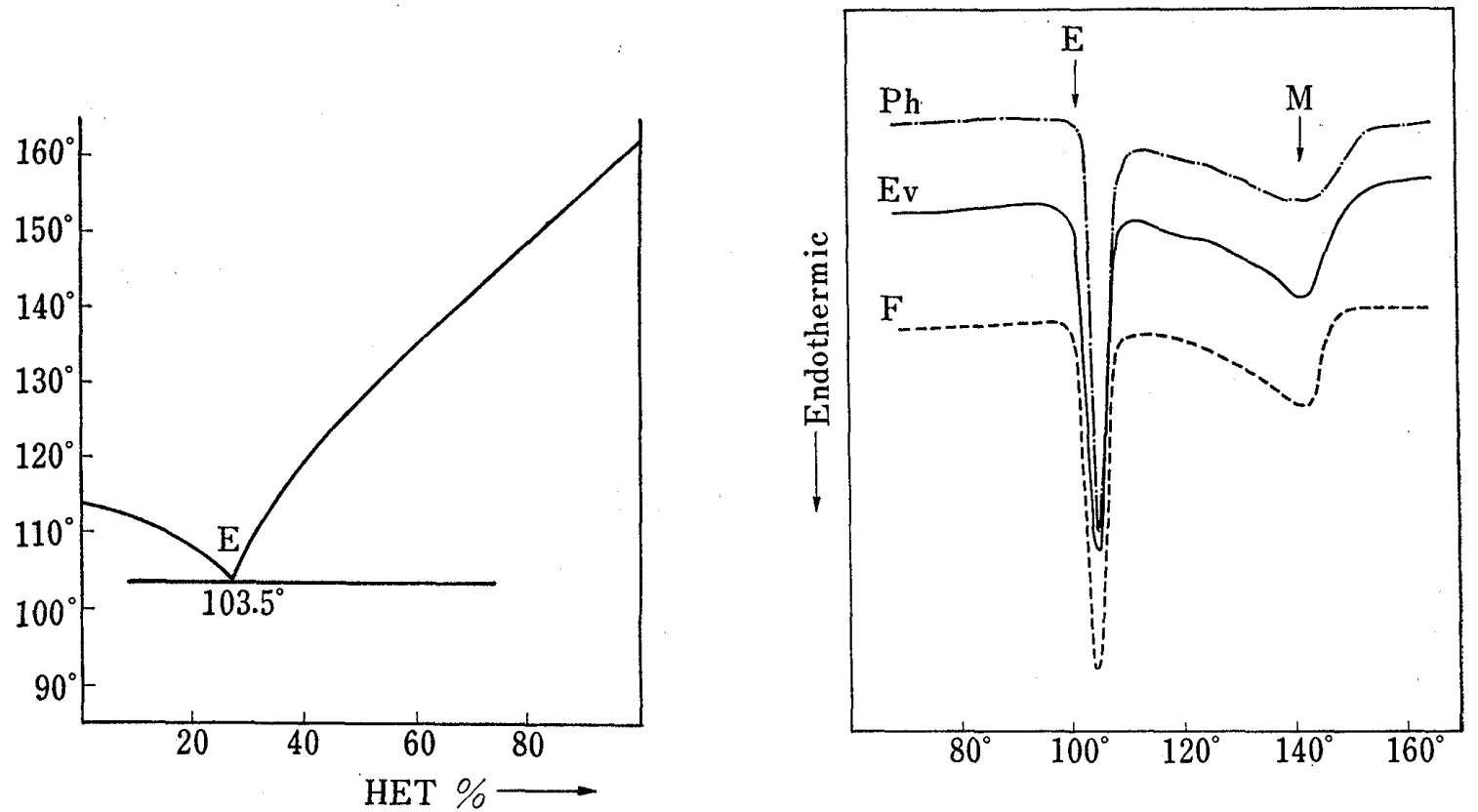

Fig. 2. Phase Diagram and DSC Curves of the System of Acetanilide and 7-Hydroxyethyltheophylline
[A] Phase Diagram
HET: 7-hydroxyethyltheophylline E : eutectic point

[B] DSC Curves for Various Kinds of Mixtures (HET 70.0\%)

Ph: physical mixt., $10.1 \mathrm{mg}$

Ev: evaporated mixt., $9.5 \mathrm{mg}$, from acetone soln.

$\mathrm{F}$ : fused mixt., $9.1 \mathrm{mg}$

scan speed: $16^{\circ} / \mathrm{min}$; range: 16

M : melting point
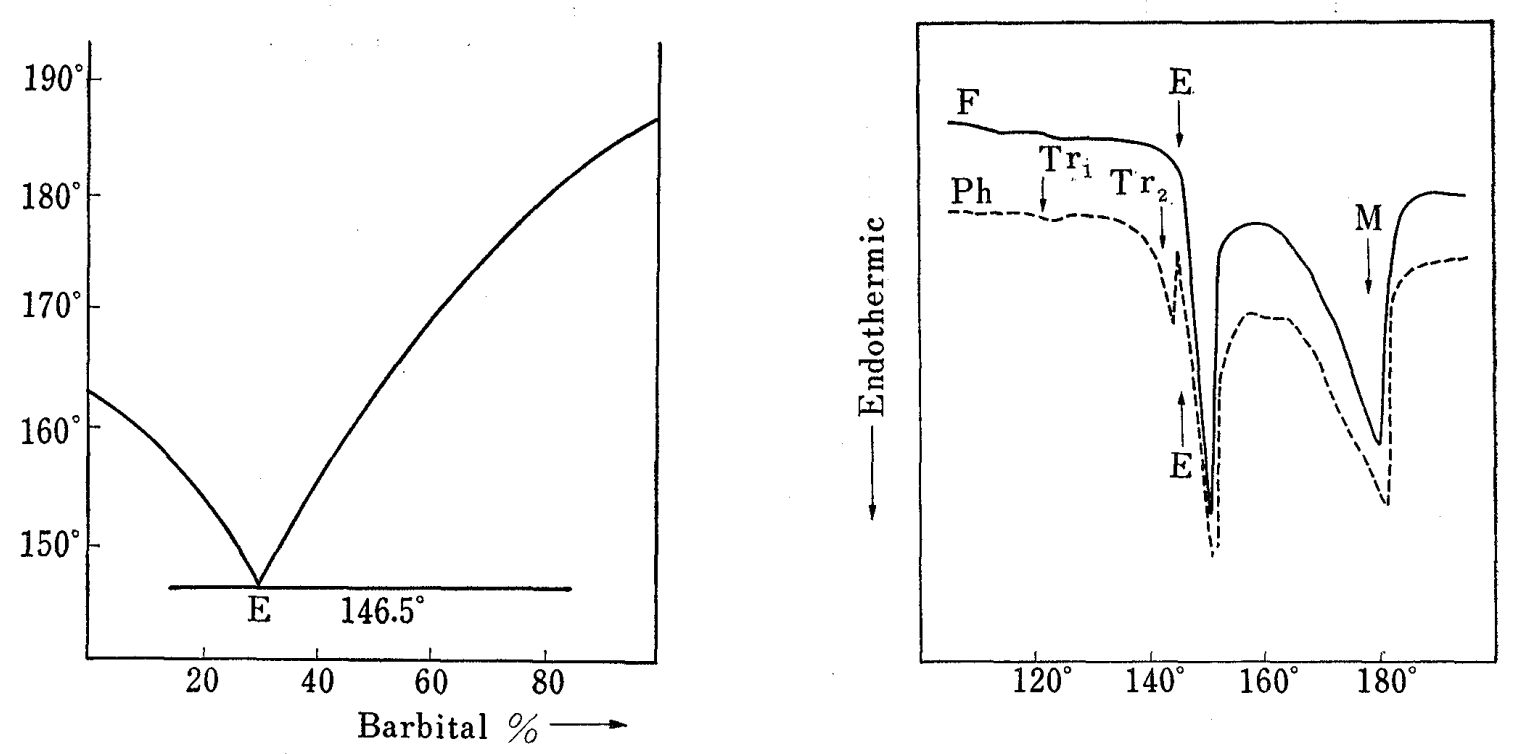

Fig. 3. Phase Diagram and DTA Curves of the System of Sulfanilamide ( $\beta$-Form) and Barbital

[A] Phase Diagram

[B] DTA Curves for Two Kinds of Mixtures (Barbital 80.0\%)

$\mathrm{Ph}, \mathrm{F}: 45 \mathrm{mg}$ of each

heating rate: $1.4^{\circ} / \mathrm{min}$; range: $25 \mathrm{mV}$

$\mathrm{Tr}_{1}$ : transition point of $\beta$-sulfanilamide

$\mathrm{Tr}_{2}$ : transition point of barbital 
sequence of appearance of these characteristic peaks, the curves are classified into two types as the following.

Type I. Consecutive Appearance of Endothermic and Exothermic Peaks due to the Metastable Eutectic Fusion and Molecular Compound Formation

The DTA and DSC curves of this type are most usual and several representative examples are shown in Fig. 4-10. Molecular compound formation is indicated by both the endothermic
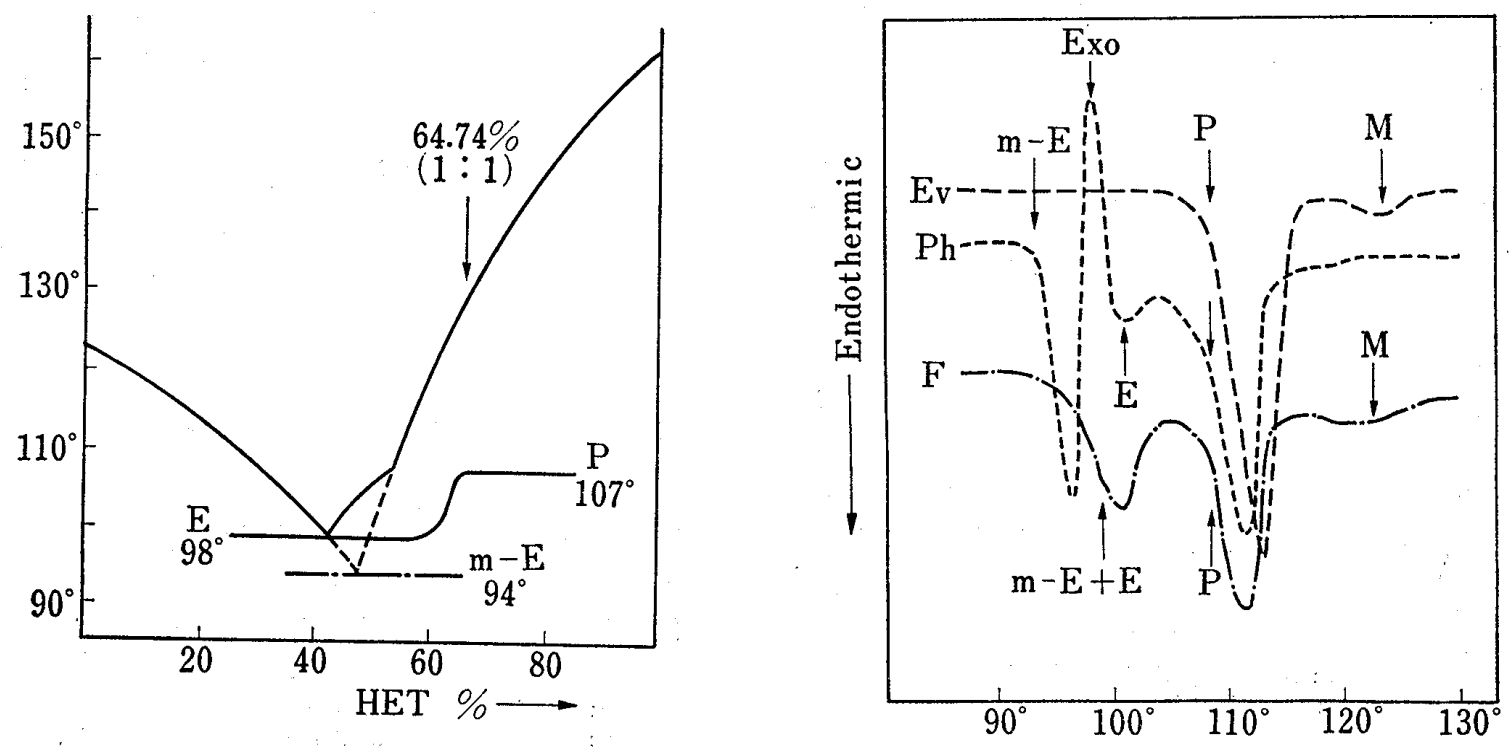

Fig. 4. Phase Diagram and DTA Curves of the System of Benzoic Acid and 7-Hydroxyethyltheophylline

[A] Phase Diagram

E : normal eutectic point

$\mathrm{m}-\mathrm{E}$ : metastable eutectic point

$P \quad$ : peritectic point

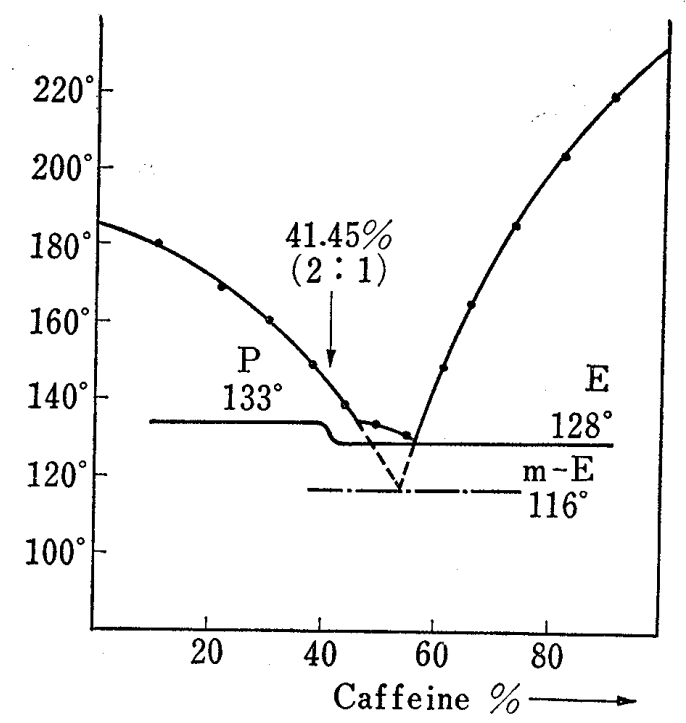

[B] DTA Curves for Various Kinds of Mixtures (HET 64.7\%, Equimolar Ratio) $\mathrm{Ph}: 62 \mathrm{mg}$; Ev: $66 \mathrm{mg}$, from acetone soln. $\mathrm{F}: 62 \mathrm{mg}$, solidified at about $50^{\circ}$ heating rate: $1.0^{\circ} / \mathrm{min}$; range: $25 \mathrm{mV}$

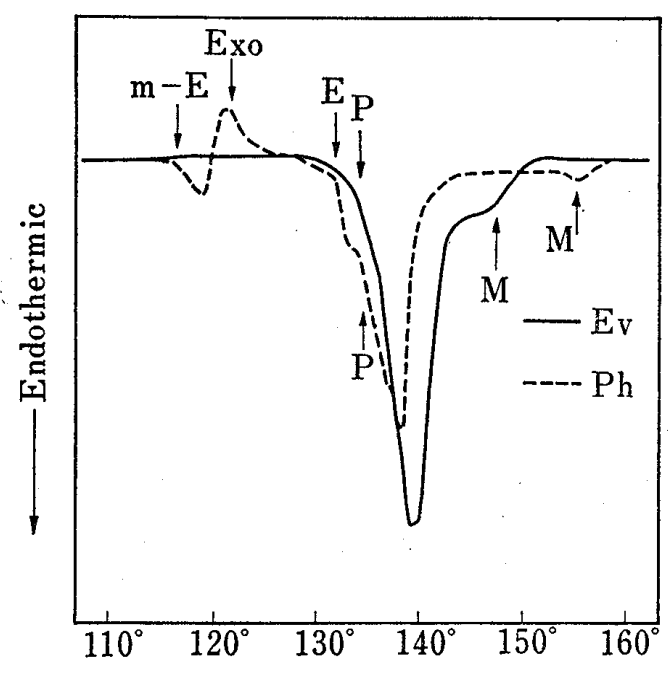

Fig. 5. Phase Diagram and DTA Curves of the System of $p$-Aminobenzoic Acid and Caffeine

[A] Phase Diagram
[B] DTA Curves for Two Kinds of Mixtures (Caffeine $38.3 \%$ )

$\mathrm{Ph}: 81 \mathrm{mg}$, intimately mixed Ev: $93 \mathrm{mg}$, from acetone soln. heating rate: $1.0 \% \mathrm{~min}$; range: $25 \mathrm{mV}$ 

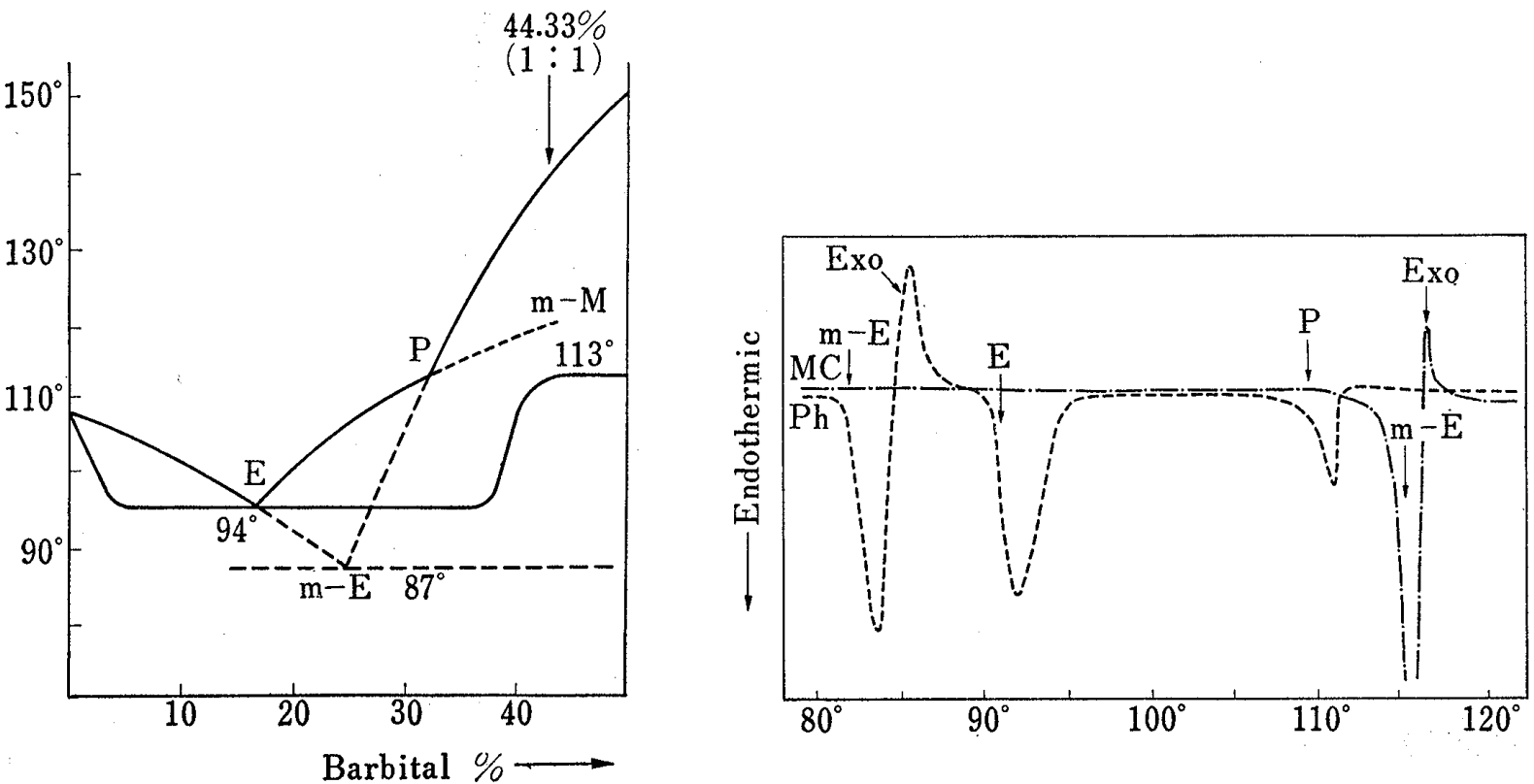

Fig. 6. Phase Diagram and DSC Curves of the System of Aminopyrine and Barbital

[A] Phase Diagram

$\mathrm{m}-\mathrm{M}$ : metastable melting point
[B] DSC Curves for the Molecular Compound and the Corresponding Physical Mixture (Barbital $44.33 \%$ )

MC: mol. compd., $10.00 \mathrm{mg}$, isolated from $\mathrm{H}_{2} \mathrm{O}$ $\mathrm{Ph}: 10.00 \mathrm{mg}$, intimately mixed scan speed: $2^{\circ} / \mathrm{min}$; range: 8

The exothermic peak of the molecular compound is caused by the solidification of barbital from the metastable melt.
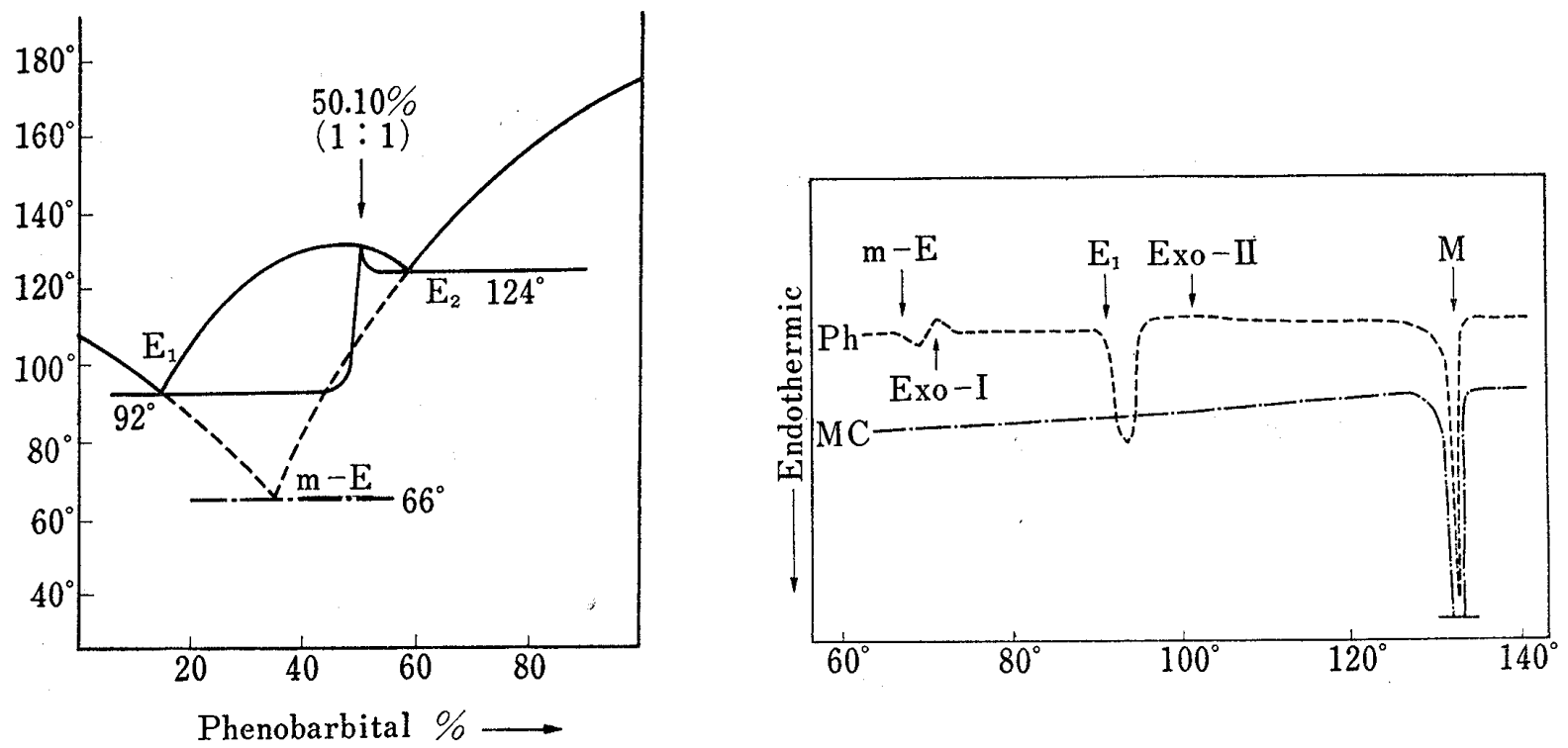

Fig. 7. Phase Diagram and DSC Curves of the System of Aminopyrine and Phenobarbital

\section{[A] Phase Diagram}

$E_{1}$ : the first eutectic point

$E_{2}$ : the second eutectic point
[B] DSC Curves for the Molecular Compound and the Corresponding Physical Mixture

(Phenobarbital $50.10 \%$, Equimolar Ratio)

MC: $10.00 \mathrm{mg}$ ( 0.0216 millimole), isolated from $\mathrm{H}_{2} \mathrm{O}$

$\mathrm{Ph}: 10.00 \mathrm{mg}$, intimately mixed

scan speed: $2^{\circ} / \mathrm{min}$; range: 8

Exo-II: Molecular compound formation and solidification occur after the first eutectic point (In respect of the total composition of the sample, fusion at $\mathrm{E}_{1}$ is also metastable.). 

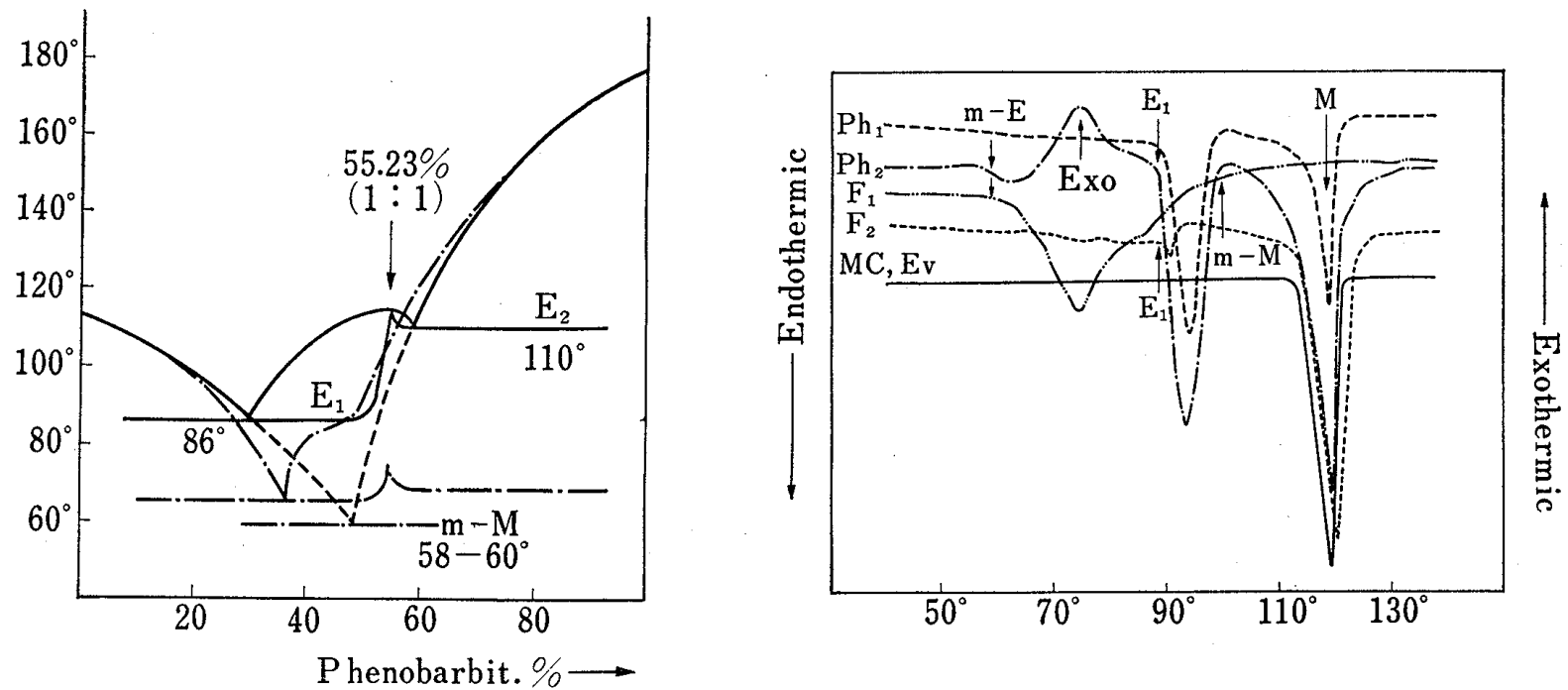

Fig. 8. Phase Diagram and DTA Curves of the System of Antipyrine and Phenobarbital

[A] Phase Diagram

- - - diagram reported by Pfeifer and Seydel diagram corrected by the authors

The metastable eutectic point was mistaken by Pfeifer and Seydel for the eutectic and peritectic points.
[B] DTA Curves for the Molecular Compound and Various Kinds of Mixtures (Phenobarbital 55.2\%, Equimolar Ratio) $\mathrm{Ph}_{1}$ : intimately mixed; $\mathrm{Ph}_{2}$ : lightly mixed $\mathrm{F}_{1}$ : solidified below $55^{\circ} ; \mathrm{F}_{2}$ : solidified at about $70^{\circ}$ $\mathrm{Ev}$ : from $\mathrm{MeOH}$ soln.; MC: isolated from $\mathrm{H}_{2} \mathrm{O}$ sample amount: $40-70 \mathrm{mg}$ heating rate: $1.0-2.0^{\circ} / \mathrm{min}$; range: $25 \mathrm{mV}$ $\mathrm{m}-\mathrm{M}\left(\right.$ Curve $\left.\mathrm{F}_{1}\right)$ : determined visually
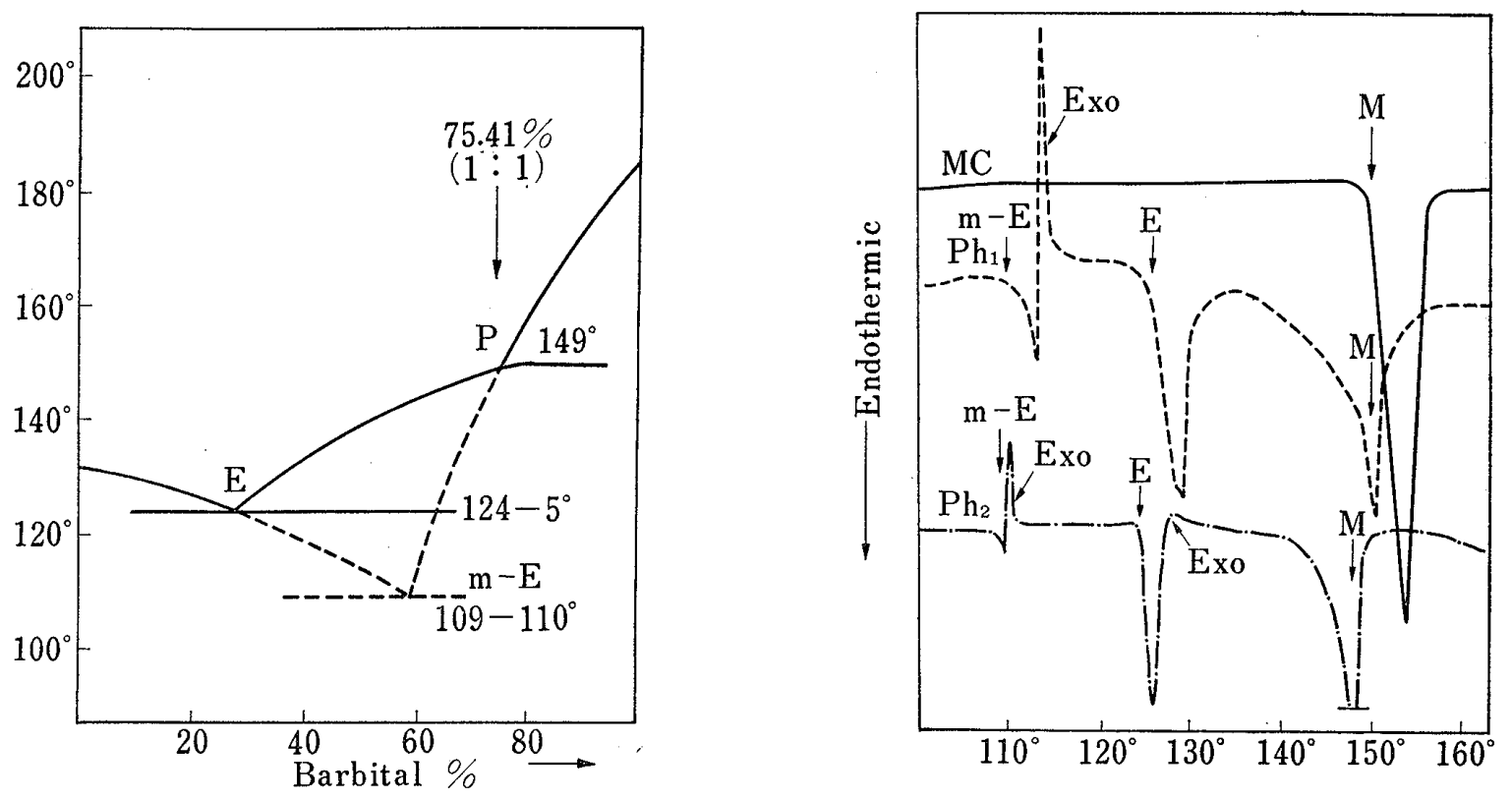

Fig. 9. Phase Diagram and DTA and DSC Curves of the System of Urea and Barbital

[A] Phase Diagram
[B] DTA and DSC Curves for the Molecular Compound and the Corresponding Physical Mixtures (Barbital 75.4\%, Equimolar Ratio)

DTA: MC : $43 \mathrm{mg}$, isolated from $n$-PrOH $\mathrm{Ph}_{1}: 44 \mathrm{mg}$, intimately mixed heating rate: $1.5^{\circ} / \mathrm{min}$; range: $25 \mathrm{mV}$

DSC : $\mathrm{Ph}_{\mathrm{z}}: 15.0 \mathrm{mg}$, intimately mixed scan speed: $2 \% \mathrm{~min}$; range: 8 

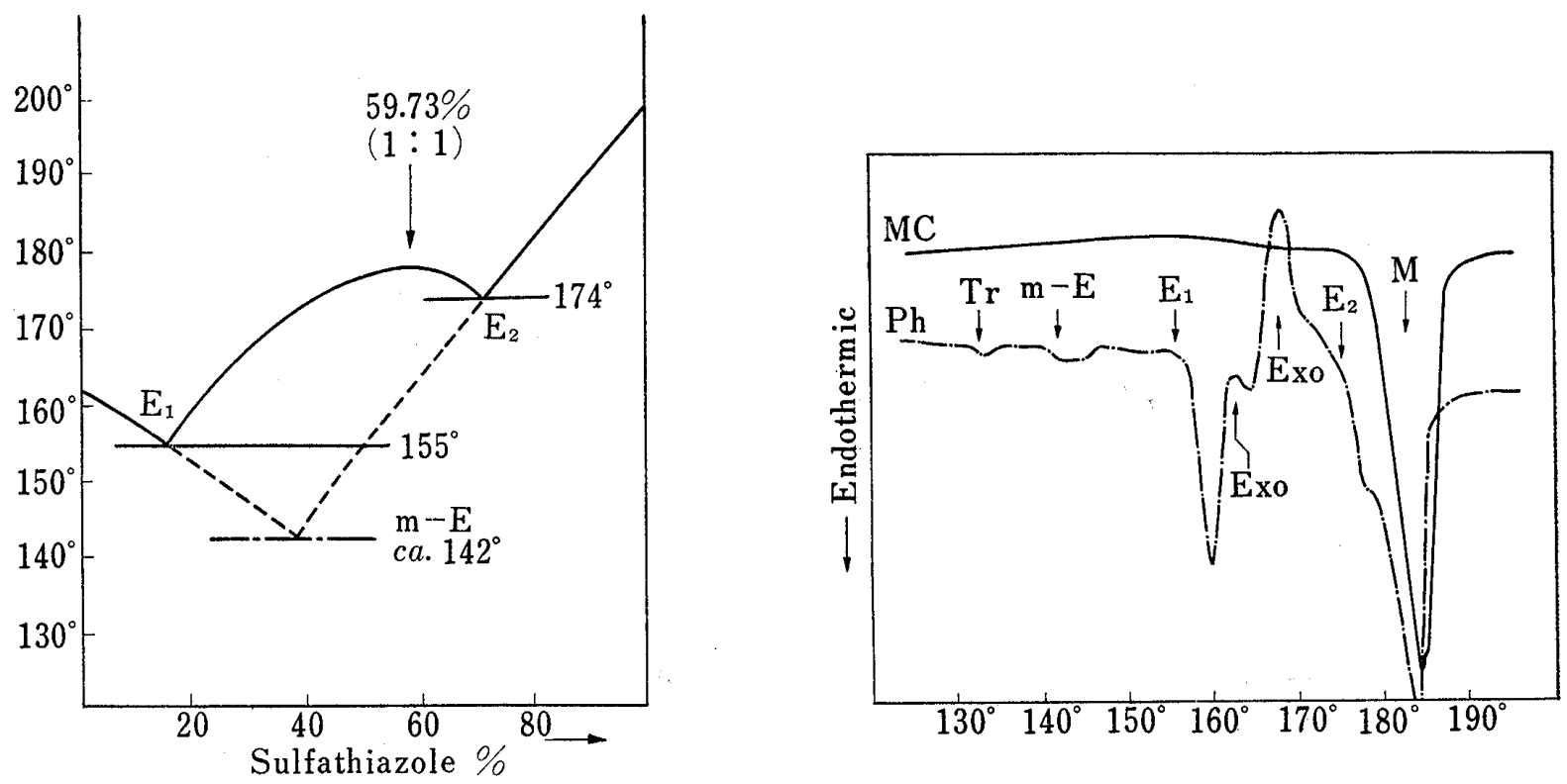

Fig. 10. Phase Diagram and DTA Curves of the System of Sulfanilamide and Sulfathiazole

[A] Phase Diagram

[B] DTA Curves for the Molecular Compound and the Corresponding Physical Mixture (Sulfathiazole 59.7\% Equimolar Ratio) MC: $52 \mathrm{mg}$, isolated from $\mathrm{MeOH}$ $\mathrm{Ph}: 51 \mathrm{mg}$, lightly mixed

heating rate: $1.5 \% \mathrm{~min}$ : range: $25 \mathrm{mV}$ $\operatorname{Tr}$ : transition point of sulfathiazole

and the exothermic peak. The rapid rise of the curve from the bottom of the endothermic peak to the summit of the exothermic one will be resulted by overlapping of opposite heat effects. As seen in the curve for the fused mixture of benzoic acid and 7-hydroxyethyltheophylline (Fig. 4 ), if the difference between the metastable and the normal eutectic points is small and the molecular compound formation and solidification occur gradually, the total heat effect is shown by a broad endothermic peak at about $94-105^{\circ}$, which is absent in the curve for the stable mixture. The fact that the normal eutectic fusion arises even in the curve of the physical mixture of which composition is equal to or greater than the combining ratio of the compound will be explained by the discussion in the previous paper. ${ }^{4)}$

\section{Type II. Consecutive Appearance of Endothermic and Exothermic Peaks due to the Normal Eutectic Fusion and Molecular Compound Formation}

Distinct evidence for the compound formation is shown by the exothermic peak after the endothermic one corresponding to the normal eutectic fusion (Fig. 11, 12). Absence of the peak for the metastable eutectic liquefaction can be explained by the fact that the metastable eutectic fusion takes place only slightly at a temperature much lower than the range of measurement or by the fact that the molecular compound is a little formed as the solid phase reaction during trituration of the solid components. In either case, direct contact between particles of both components is prevented by the film of the molecular compound; consequently, the compound is formed almost entirely in the normal eutectic liquid.

The col or the shoulder in the exothermic peak (Fig. 10,11) indicates that two different exothermic reactions occur succesively. The preceeding one is thought to be due to the compound formation, while the following will be attributed to the solidification of the eutectic melt which is also metastable with respect to the composition of the whole sample in the cell. In the first type of curves for the physical mixture, two separate exothermic peaks appears occasionally (Fig. 7, 9). Such a pattern of the curve will be considered as a composite of the type I and II. 


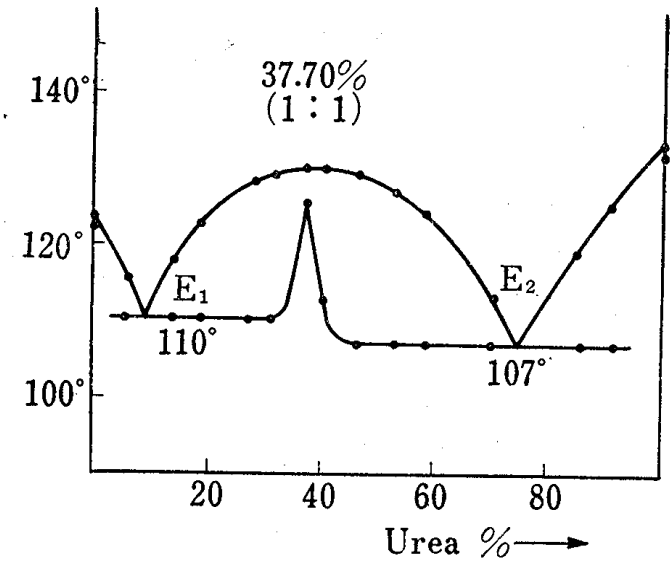

Fig. 11. Phase Diagram and DTA Curves of the System of Succinimide and Urea

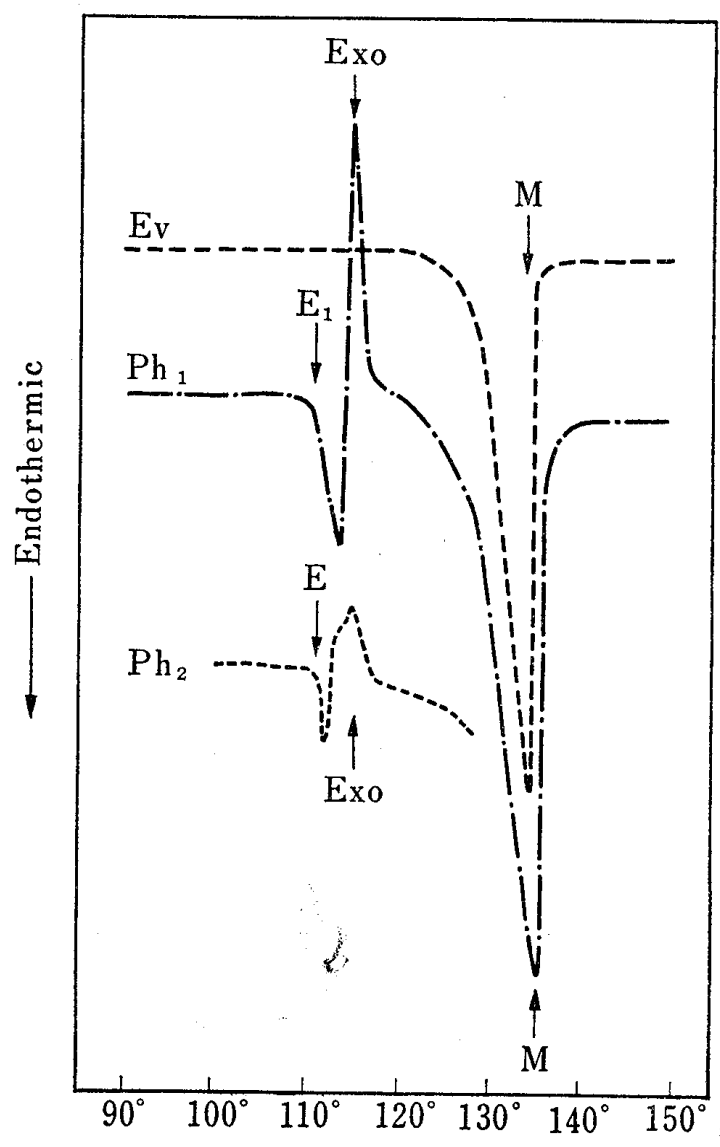

[A] Phase Diagram

[B] DTA Curves for Two Kinds of Mixtures

(Urea 37.7\%, Equimolar Ratio)

$\mathrm{Ph}_{1}: 45.0 \mathrm{mg}$, lightly mixed

$\mathrm{Ph}_{2}: 60.5 \mathrm{mg}$, intimatly mixed

$\mathrm{Ev}: 55.0 \mathrm{mg}$, from acetone-MeOH soln.

heating rate: $\mathrm{Ph}_{1}: 1.5^{\circ} / \mathrm{min}, \mathrm{Ph}_{2}: 1.0^{\circ} / \mathrm{min}$

range: $25 \mathrm{mV}$

\section{Conclusion}

From these results, it is clear that in the DTA or DSC curve for the physical mixture, appearance of peaks below and above the base line give a powerful evidence for the molecular compound formation. Although in a few cases, the exothermic peak disappears or appears beneath the base line, formation of the compound can be detected by comparing the curve with the one for the evaporated mixture, since the latter solidifies usually in the stable equilibrium state. Similarly, peaks due to the polymorphic transition of one or two components can be assigned by the curve of the component itself, and the confusion by such peaks will be mostly avoided. Thus, detection of the molecular compound between organic materials will be done by applying DTA or DSC once or twice.

Also, determination of the heat of formation of the molecular compound is possible. The heat is calculated by the following equation,

$$
\Delta H=\frac{\Delta S \times[\text { range setting] }}{\text { [chart speed][chart width] }} \times \frac{\text { [mol. wt. of the molecular compound] }}{\text { [moles of the molecular compound in the sample] }}
$$

where $\Delta H$ is the heat of formation and $\Delta S$ is the difference between the total surface area of DSC peaks for the isolated molecular compound or its physical mixture with one component and the peak area for the corresponding physical mixture of both components. For the 

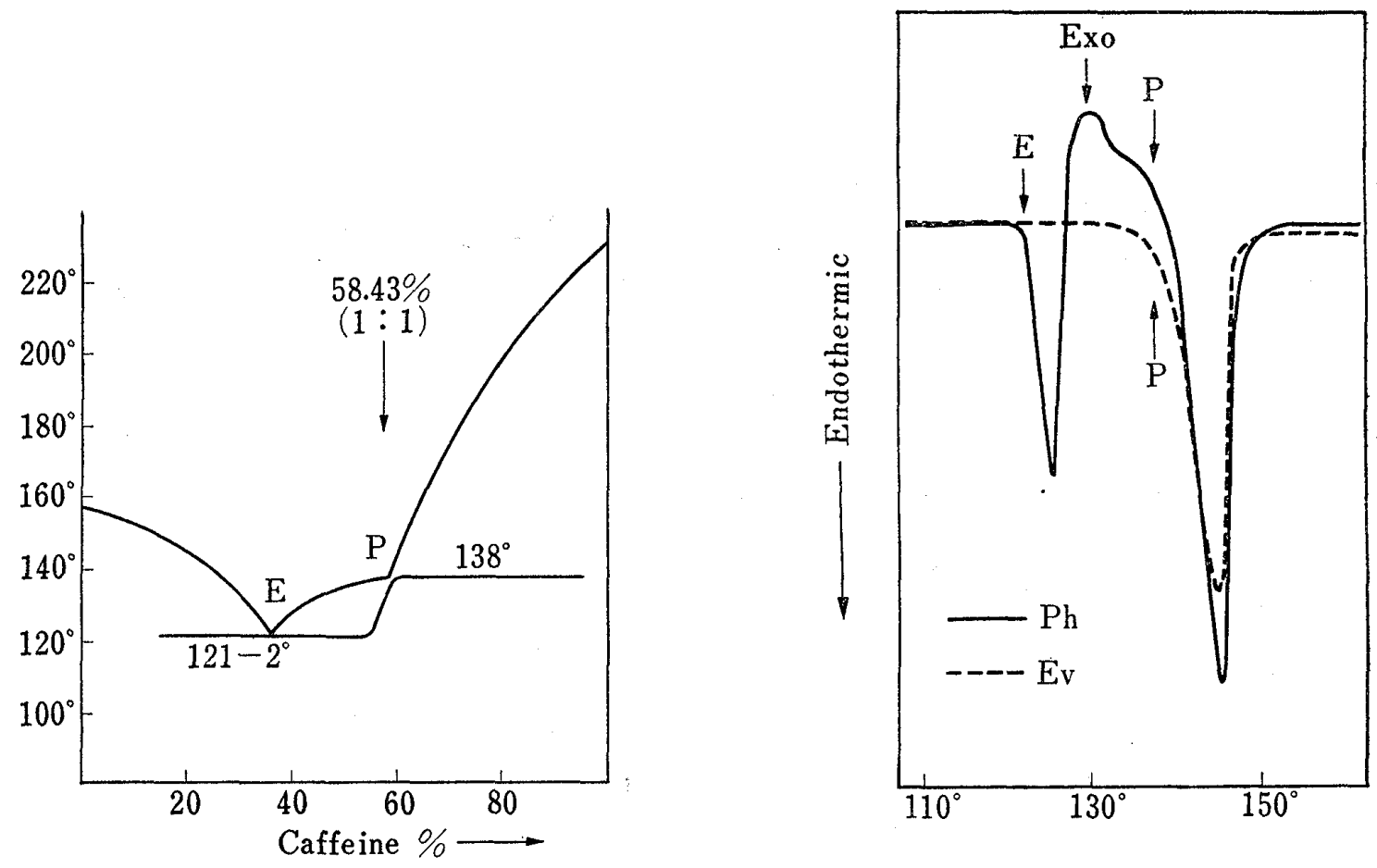

Fig. 12. Phase Diagram and DTA Curves of the System of Salicylic Acid and Caffeine

[A] Phase Diagram

[B] DTA Curves for Two Kinds of Mixtures (Caffeine 65.0\%)

$\mathrm{Ph}: 43.5 \mathrm{mg}$, lightly mixed

Ev: $42.2 \mathrm{mg}$, from acetone soln.

heating rate: $1.5^{\circ} / \mathrm{min}$

range: $25 \mathrm{mV}$

system of aminopyrine and phenobarbital, the heat of compound formation was found to be $-1.0 \pm 0.1 \mathrm{kcal} / \mathrm{mole}$. Details on this subject will be reported later.

Although it has been known that as a special kind of physical incompatibility, ${ }^{6}$ ) a mixture of solid ingredients once become pasty or liquefies by trituration but it changes into a solid mass on standing overnight, the cause of the phenomenon has not hitherto been investigated. As one of the authros previously pointed out,7) such an incompatibility in dispensing will no doubt be attributed to the metastable eutectic liquefaction found in the mixture of which components react to form a molecular compound. The phenomenon will also cause difficulties in the production of solid pharmaceutical preparations, since the heat evolved during mixing, sieving, and compressing will sometimes rise the temperature higher than the metastable eutectic point. Even if the preparation can be formed, pharmaceutical properties of the final product will depend on the conditions in the manufacturing processes.

6) Y. Sugii and N. Nishioji, "Yakuhin Haigokinki (Drug Incompatibility)," Nanzando, 1963.

7) K. Sekiguchi, Yakugaku Zasshi, 81, 670 (1961). 